\title{
Guest editorial: Interaction in immersive experiences
}

\author{
Harry Agius $^{1}$ [D $\cdot$ Damon Daylamani-Zad ${ }^{1} \mathbb{D}$
}

Accepted: 13 July 2021 / Published online: 3 August 2021

(c) The Author(s), under exclusive licence to Springer Science+Business Media, LLC, part of Springer Nature 2021

Immersive technologies are now highly accessible in the consumer market and rapidly becoming part of our everyday lives. Advances in computing power, mobile devices, display technologies, and widespread high-speed networks, coupled with increasing research and development in digital immersive media and artificial intelligence (AI) has led to novel uses and applications, and development of advanced techniques for immersing users into digital media experiences and engaging them in innovative ways via increasingly sophisticated interaction methods.

Most notably, eXtended Reality (XR), which encompasses Augmented Reality (AR), Virtual Reality (VR) and Mixed Reality (MR), has become accessible and affordable to consumers, facilitating a wide range of opportunities for immersive interactions [3]. XR on mobile devices has provided new experiences for interacting with and interpreting the world around us, with games such as Pokemon Go, Ingress Prime and Knightfall: AR, educational experiences such as $B B C$ Civilisations $A R$, shopping support experiences such as IKEA Place, and practical everyday tools such as Google Lens, Translate and Maps AR.

More recently, virtual production, centred around real-time interaction on production sets that combine physical and digital worlds, takes advantage of the visualisation capabilities of MR and VR. The use of XR and virtual interactions has revolutionised production and shifts the pipelines and creative workflows of filmmaking [5]. Novel dramatic experiences such as Draw Me Close and All Kinds of Limbo at the Immersive Storytelling Studio of the National Theatre in the UK take advantage of immersive technologies. Immersive experiences such as The Tempest created by the Royal Shakespeare Company in collaboration with Intel and Imaginarium Studios also demonstrate the effective role AR can play in bringing about immersive theatre.

Meanwhile, advances in 5G communications have shown great potential in live rendering and rapid delivery of high-quality immersive content [13] for creating location-based, personalised experiences using cloud-based services and machine learning approaches [9], such as NVIDIA's CloudXR for streaming intensive graphics for XR and GridRaster's AIenabled, immersive MR platform, and assisted in creating more meaningful and engaging experiences, such as PopStic VR and Epic's Apollo 11: Mission AR on Microsoft

Harry Agius

Harry.Agius@brunel.ac.uk

Damon Daylamani-Zad

Damon.Daylamani-Zad@brunel.ac.uk

1 Digital Media, Brunel Design School, College of Engineering, Design and Physical Sciences, Brunel University London, Kingston Lane, Uxbridge, London UB8 3PH, UK 
HoloLens. Microsoft Mesh is also disrupting how we share experiences, collaborate, communicate, and recognise presence by disrupting our interactions with real and virtual worlds and bringing digital intelligence to the real world.

This special issue brings together recent state-of-the-art advances in immersive experiences. It is formed of 12 papers from a collection of international researchers spanning 13 countries: Australia, China, Cyprus, Czech Republic, Denmark, Hungary, Poland, Portugal, Republic of Korea, Romania, Spain, UK, and USA. As such, it provides a useful snapshot of global progress in immersive experiences.

The first four papers deal to varying extents with content presentation in immersive experiences. In the opening paper, Pamparău and Vatavu [11] tackle the issue of augmented and mediated visual content, focusing on providing flexibility in the configuration and customisation of AR features and functionalities. To this end, they propose FlexiSee, an opensource application for smart eyewear devices that enables the user to specify and apply custom visual filters, and FlexiSee-DS, a design space that enables a range of flexible design options to be specified within mediation and augmentation, user roles, and control modalities. The next paper by Doukianou, Daylamani-Zad and O'Loingsigh [2] explores AR from the perspective of business presentation content. They propose the design and development of an AR presentation application that supports animated and interactive infographics and, through a large-scale study, found that their approach provides enhanced usability, audience engagement and communication effectiveness when compared with traditional presentations. The next paper by Hughes and Montagud [4] considers $360^{\circ}$ video players and their accessibility. After a comprehensive evaluation of current web-based and standalone $360^{\circ}$ video players, highlighting their shortfalls, they propose the open-source web-based and personalised ImAc player, which sits within the modular end-to-end ImAc platform that allows the integration of immersive and accessible content in current broadcast-related services. Continuing the concern with $360^{\circ}$ video, the paper by Marques, Vairinhos and Almeida [10] explores whether VR $360^{\circ}$ video content can provide greater immersion for viewers. To determine this, they produced suspenseful content as a short-film adaptation of Edgar Allan Poe's The Tell-Tale Heart in conventional 2D, non-VR $360^{\circ}$, and VR $360^{\circ}$ formats, and compared how participants reacted to the three versions. Their results indicate that VR $360^{\circ}$ facilitates perceptual immersion but negatively impacts upon narrative immersion.

The next three papers in this special issue focus on emerging input and interaction approaches appropriate for immersive experiences. Tadeja et al. [14] propose an approach for bimanual gestural interaction in VR coupled with gaze-tracking. The feasibility of the approach is tested in an engineering case study to support the manipulation and measurement of complex photogrammetric 3D structure models. The expert participants in the study reported favourably on the approach and two design implications were revealed, concerning model manipulation and measurement. Gestures are also investigated in the next paper by Wang et al. [16], which proposes an MR remote collaboration system that enables a remote expert in VR to provide instructions based on 3D gesture and CAD models for a local worker who uses AR to see these instructions. A user study revealed the potential of the approach to improve performance time and user experience when remotely collaborating on an assembly task. Considering physical immersion experiences, Čejka, Chmelík and Liarokapis [1] propose an approach to improve typing on a smartphone while underwater. Their Fan 2 keyboard exploits the use of tilting to provide input by utilising orientation sensors. It uses two steps to select each letter (selecting a group and then the required letter within the group) and was found to provide quantitative and qualitative advantages over other tilting keyboard methods. 
Two of the papers in this issue address immersive authoring issues. The paper by Takacs and Vincze [15] proposes a pipeline incorporating a parallel deep neural network to support the automated authoring of immersive $360^{\circ} \mathrm{XR}$ experiences. Their approach is demonstrated extensively through four case studies: a virtual play-along music experience, a set of $6 \mathrm{DoF}$ representations created from monoscopic stitched VR footage, a series of interactive virtual actors placed within a $360^{\circ}$ scene, and the conversion of a spherical film into a VR experience. In contrast, the paper by Kim et al. [6] proposes the RealityBrush system that enables designers to create realistic virtual objects by capturing and utilising the kinetic properties of physical objects. Key to the system is a rod-like handheld device that is equipped with a depth camera and a force sensor. Technical and user evaluations discovered that the system can successfully extract the stiffness and motion resistance of everyday objects and was reported on favourably by users.

The final three papers look at the application of immersive technologies for serious games and learning. In the paper by Park et al. [12], a smart mirror is used to implement a personalised fitness training system based on a proposed "Apart Yet Together" schema. In this way, users can copy the exercise postures of a customised virtual trainer synchronously and receive coaching through real-time motion correction. Careful evaluation revealed a positive impact on user satisfaction and experience effectiveness. The next paper by Lau and Agius [7] proposes a framework for developing cognitively and physically rehabilitative immersive serious games for patients diagnosed with MCI (mild cognitive impairment). The framework is demonstrated via an immersive gestural serious game that takes players through several therapeutic scenarios and tasks in conjunction with their occupational therapist. Evaluation revealed that the framework offers a comprehensive approach not currently provided by other rehabilitative frameworks, while the game provides an increase in patient support over traditional rehabilitative training. The final paper by Lee et al. [8] brings the special issue to a close by investigating discipline and guidance for visual engagement in immersive virtual learning environments. In their experiment, users are required to manipulate a virtual machine with multiple types of controllers and are supported by a video lecture of the task which is shown on a virtual screen in the immersive environment. Visual stimuli are eliminated and appended to facilitate discipline and guidance, respectively. The results revealed that the visual engagement approach impacts usability of learning and they conclude that guidance would be beneficial when the user's engagement needs to be maintained while discipline would be beneficial for training that demands intense concentration.

\section{References}

1. Čejka J, Chmelík J, Liarokapis F (2021) Exploring tilting methods for typing under water. Multimedia Tools and Applications. https://doi.org/10.1007/s11042-020-09305-7

2. Doukianou S, Daylamani-Zad D, O'Loingsigh K (2021) Implementing an augmented reality and animated infographics application for presentations: effect on audience engagement and efficacy of communication. Multimedia Tools and Applications. https://doi.org/10.1007/s11042-021-10963-4

3. Doukianou S, Daylamani-Zad D, Paraskevopoulos I (2020) Beyond Virtual Museums: Adopting Serious Games and Extended Reality (XR) for User-Centred Cultural Experiences. In: Liarokapis F, Voulodimos A, Doulamis N, Doulamis A (eds) Visual Computing for Cultural Heritage. Springer, Cham, Switzerland, pp 283-299, https://doi.org/10.1007/978-3-030-37191-3_15

4. Hughes CJ, Montagud M (2021) Accessibility in $360^{\circ}$ video players. Multimedia Tools and Applications. https://doi.org/10.1007/s11042-020-10088-0 
5. Ilmaranta K (2020) Cinematic Space in Virtual Production. In: De Paolis L, Bourdot P (eds) Augmented Reality, Virtual Reality, and Computer Graphics. AVR 2020. Lecture Notes in Computer Science, Vol. 12243. Springer, Cham, Switzerland, pp 321-332, https://doi.org/10.1007/978-3-03058468-9_23

6. Kim H, Hong S, Kim J et al (2021) RealityBrush: an AR authoring system that captures and utilizes kinetic properties of everyday objects. Multimedia Tools and Applications. https://doi.org/10.1007/ s11042-020-09332-4

7. Lau SYJ, Agius H (2021) A framework and immersive serious game for mild cognitive impairment. Multimedia Tools and Applications. https://doi.org/10.1007/s11042-021-11042-4

8. Lee H, Jung J, Lee HK, Yang HS (2021) Discipline vs guidance: comparison of visual engagement approaches in immersive virtual environments. Multimedia Tools and Applications. https://doi.org/10. 1007/s11042-020-10267-z

9. Liubogoshchev M, Ragimova K, Lyakhov A et al (2021) Adaptive Cloud-Based Extended Reality: Modeling and Optimization. IEEE Access 9:35287-35299. https://doi.org/10.1109/ACCESS.2021. 3062555

10. Marques T, Vairinhos M, Almeida P (2021) VR $360^{\circ}$ and its impact on the immersion of viewers of suspense AV content. Multimedia Tools and Applications. https://doi.org/10.1007/s11042-021-11067-9

11. Pamparău C, Vatavu RD (2021) FlexiSee: flexible configuration, customization, and control of mediated and augmented vision for users of smart eyewear devices. Multimedia Tools and Applications. https://doi.org/10.1007/s11042-020-10164-5

12. Park HS, Lee GA, Seo BK, Billinghurst M (2021) User experience design for a smart-mirror-based personalized training system. Multimedia Tools and Applications. https://doi.org/10.1007/s11042-020-10148-5

13. Schmoll RS, Pandi S, Braun PJ, Fitzek FHP (2018) Demonstration of VR / AR offloading to Mobile Edge Cloud for low latency 5G gaming application. In: CCNC 2018 - 15th IEEE Annual Consumer Communications and Networking Conference. IEEE, Las Vegas, NV, USA, pp 1-3, https://doi.org/10. 1109/CCNC.2018.8319323

14. Tadeja SK, Lu Y, Rydlewicz M et al (2021) Exploring gestural input for engineering surveys of reallife structures in virtual reality using photogrammetric 3D models. Multimedia Tools and Applications. https://doi.org/10.1007/s11042-021-10520-z

15. Takacs B, Vincze Z (2021) Deep authoring - an AI Tool set for creating immersive MultiMedia experiences. Multimedia Tools and Applications. https://doi.org/10.1007/s11042-020-10275-Z

16. Wang P, Bai X, Billinghurst $M$ et al (2021) 3DGAM: using 3D gesture and CAD models for training on mixed reality remote collaboration. Multimedia Tools and Applications. https://doi.org/10.1007/ s11042-020-09731-7

Publisher's Note Springer Nature remains neutral with regard to jurisdictional claims in published maps and institutional affiliations. 